\title{
Procedimientos tributarios para el manejo y control del impuesto al valor agregado (IVA) en la industria farmacéutica
}

\section{Tax procedures for the management and control of value added tax (VAT) in the pharmaceutical industry}

\author{
Gabriela Estefanía Cabrera Ramón \\ gcabrerar@psg.ucacue.edu.ec \\ Universidad Católica de Cuenca, Cuenca \\ Ecuador \\ https://orcid.org/0000-0002-2301-4726 \\ Cecilia Ivonne Narváez Zurita \\ inarvaez@ucacue.edu.ec \\ Universidad Católica de Cuenca, Cuenca \\ Ecuador \\ https://orcid.org/0000-0002-7437-9880 \\ Juan Carlos Erazo Álvarez \\ jcerazo@ucacue.edu.ec \\ Universidad Católica de Cuenca, Cuenca \\ Ecuador \\ https://orcid.org/0000-0001-6480-2270
}

Recibido: 1 de septiembre de 2019

Aprobado: 30 de septiembre de 2019

\section{RESUMEN}

El presente artículo tiene como objetivo explorar las necesidades específicas de estandarización en la gestión tributaria para diseñar y diagramar lineamientos que procuren la gestión tributaria en esta empresa. El tipo de investigación fue descriptiva dado que se expusieron características propias de los procedimientos tributarios para el tratamiento del IVA en una empresa pública. Como unidad de analisis se consideró a la Empresa FARMASOL EP., y como universo de estudio a cuatro actores clave de la empresa: Gerente, Subgerente Administrativo Financiero, Contador y Tesorero. se concluye que los tributos juegan un papel vital para las economías de las personas, las organizaciones y los países. De estos, el pago y administración tributaria del IVA puede impactar positiva o negativamente a la liquidez y utilidades de las empresas. Esto 
dependiendo de si la gestión tributaria se realiza mediante lineamientos o procedimientos tributarios acordes a la ley.

Descriptores: Tributos; Impuestos; Impuesto al valor agregado; Procedimientos tributarios.

\section{ABSTRACT}

This article aims to explore the specific needs of tax management standardization to design and diagram guidelines that seek tax management in this company. The type of investigation was descriptive given that characteristics of the tax procedures for the treatment of VAT in a public company were presented. The FARMASOL EP. Company was considered as an analysis unit, and as a study universe four key actors of the company: Manager, Financial Administrative Assistant Manager, Accountant and Treasurer. It is concluded that taxes play a vital role for the economies of people, organizations and countries. Of these, the payment and tax administration of VAT can positively or negatively impact the liquidity and profits of companies. This depends on whether the tax management is carried out through tax guidelines or procedures according to the law.

Descriptors: tributes; taxes; value added tax; tax procedures

\section{INTRODUCCIÓN}

Los impuestos o tributos son una de las principales fuentes de ingreso público (Larraín y Sachs, 2002). Esto es debido a su aporte significativo al ingreso fiscal nacional. De estos, el impuesto al valor agregado (IVA), es uno de los más comunes a nivel global (Paz y Cepeda, 2015). Sin embargo, se ha evidenciado que este impuesto, al ser de carácter obligatorio y recurrente, afecta directamente a la economía de los ciudadanos y de los empresarios (Vatlive, 2018). Esto se debe principalmente a las transacciones cotidianas de bienes y servicios que gravan IVA, mismas que tienen una afección directa en la liquidez y en las utilidades, además de otros riesgos relacionados.

Un sector que ha mostrado un desarrollo sostenido a nivel local e internacional, es la industria farmacéutica (ClústerFarma, 2017). Esta industria tiene un carácter social y económico con la ciudadanía debido a los bienes y servicios que se ofertan. Por otro lado, el sector público, al operar bajo la constitución nacional y estar alineado a principios 
económicos, sociales y de desarrollo sostenible, tiene especial interés por el manejo correcto de los fondos públicos y de la diseminación de información financiera. Por tal razón, Farmasol EP, una empresa Farmacéutica del sector público de Cuenca Ecuador, ha sido seleccionada.

Farmasol EP., ha tenido un crecimiento sostenido en los últimos años. Cifras indican que en promedio, la empresa inaugura 2 farmacias nuevas cada año. Además, sus estados financieros muestran utilidades, han realizado re inversiones y se evidencia un cumplimiento óptimo del Plan Operativo Anual (POA). Así mismo, es una de las empresas más importantes de la corporación municipal local con presupuestos anuales promedio de 20 millones de dólares.

El presente caso de estudio tiene dos objetivos: primero, explorar el grado de aplicación de procedimientos o lineamientos tributarios en la empresa FARMASOL EP; segundo, diseñar y diagramar procedimientos tributarios del manejo del IVA para mejorar la gestión tributaria del IVA en esta empresa.

Los impuestos o tributos son considerados como la principal fuente de ingresos públicos debido a su aporte significativo al ingreso fiscal (Vargas, 2017). Paz y Cepeda (2015), indican que estos forman una parte importante dentro del presupuesto de los gobiernos, usados en gran medida para la ejecución de obras en beneficio de la población.

Existen diversos tipos de impuestos de acuerdo al concepto de la transacción (Say, 1983): los impuestos directos son aquellos que gravan a la manifestación directa o inmediata de capacidad económica; los indirectos son los que gravan la capacidad económica que se pone de manifiesto de forma indirecta como consecuencia de la circulación de la riqueza

Uno de los tributos más comunes es el IVA por sus siglas en español. El IVA es un tributo indirecto y progresivo que se cobra sobre la transferencia de dominio de bienes y servicios (Benitez, 2016). Este impuesto se paga por cada transferencia de dominio en bienes y servicios de acuerdo con las diferentes legislaciones de cada país. Sin embargo, el peso del IVA varía en cada país. Un reporte de la BBC señala que a nivel mundial, el promedio del impuesto al valor agregado es del 15\%, mientas que en América Latina es 
del 9\% (Seitz, 2015). El autor sostiene que la tendencia actual es aumentar esta tarifa y ampliar los sectores a los cuales aplicar este tributo.

En cuanto al pago del IVA, se postula que este impuesto, al ser cancelado por el contribuyente final en la adquisición de bienes y servicios, tiene una afectación económica directa en la población (Vatlive, 2018). Incluso se sugiere que las altas tasas de impuestos tributadas para el gobierno juegan un rol importante en las crisis económicas. Martínez Prats (2019).

\section{El IVA en el Ecuador}

De acuerdo a Vargas (2017), el IVA es el impuesto que grava a la transferencia de dominio de bienes y servicios de acuerdo a la tarifa vigente en cada país. Además, el autor indica que se trata de un impuesto indirecto, es decir que grava a cada traslado de dominio o fase hasta llegar al consumidor final o sujeto pasivo del impuesto.

En cuanto al contexto de este tributo en el Ecuador, la Asamblea Constituyente Nacional (2015), en la Ley de Régimen Tributario Interno o LORTI, establece que el IVA es el tributo que grava el valor de las transferencias locales o importaciones de bienes muebles en todas sus etapas de comercialización y al valor de los servicios prestados. Además, una vez se haya pagado el IVA, las empresas podrán utilizarlo como crédito tributario según casos contemplados en la LORTI.

Además, el IVA es sujeto de declaración. Por ejemplo, este impuesto debe ser declarado y cancelado mensualmente. Esto cuando se realizan retenciones o también cuando las transacciones gravan una tarifa. También se lo realiza cada seis meses en las transacciones que graven tarifa $0 \%$. Esto aplica transferencias locales o importaciones de bienes y en la prestación de servicios (Sistema de Rentas Internas, 2018).

En el caso de transacciones locales, el precio de venta de los bienes o el valor de la prestación del servicio representa la base imponible del IVA. Aquí se incluyen tasas y otros impuestos y gastos relacionados menos descuentos y/o devoluciones. En cuanto a las importaciones, la base imponible del IVA se obtiene de valor total del CIF i.e. costo, 
seguro y flete. Esto se adiciona los aranceles, tasas, impuestos, derechos y recargos incluidos en la declaración de importación.

Actualmente, el peso del IVA en el Ecuador es del 12\%. Dicho porcentaje grava a bienes y servicios según indica la ley. En contraste, existen bienes y servicios que tienen la característica de no ser objeto de IVA según indica en los artículos 54 y 55 de la LORTI (2015): transferencias que no son objeto del impuesto y transferencias e importaciones con tarifa cero.

Por otro lado, al producir o comercializar bienes o servicios que gravan IVA, las empresas y negocios pueden acogerse al beneficio del crédito tributario. Dicho crédito es una compensación del impuesto pagado sobre bienes, servicios o insumos adquiridos y necesarios para la producción y/o comercialización de los bienes y servicios sujetos del valor del IVA. (Hidalgo, Narváez y Erazo, 2019)

Esto se debe a que el IVA opera bajo una modalidad de crédito, es decir se paga el IVA que se cobra en las ventas menos el valor del IVA pagado en compras. De esta operación se desprende el concepto de crédito tributario i.e. cuando el total del IVA pagado en compras es superior al cobrado en ventas. Este resultante positivo, de acuerdo al artículo 66 de la LORTI (2015), puede ser utilizado para compensar el impuesto a pagar del siguiente mes.

Adicionalmente, incluso cuando las organizaciones o negocios no puedan acceder al crédito tributario, el IVA pagado pude ser trasladado al precio de venta.

\section{Devoluciones, compensaciones y reembolsos}

Por otra parte, dentro de la legislación ecuatoriana también aparece el concepto de devolución del IVA por parte del Estado en casos que permita la ley (Asamblea Constituyente Nacional, 2015). Por ejemplo: devolución del IVA a proveedores directos de exportadores de bienes, devolución a entidades especificadas y devolución del IVA sobre retenciones en la fuente.

En cuanto a compensaciones del IVA, esto se detalla en el Código Tributario del Ecuador (2005). Por un lado, las compensaciones usadas como devoluciones, son ejecutadas a 
través de la supresión de una obligación tributaria con créditos líquidos reconocidos. Por otro lado, los valores reconocidos con deudas tributarias firmes que requieran ser compensados; son ejecutados mediante un acto administrativo o judicial. Mediante dicho acto que dispone se dispone la compensación por devolución de impuestos.

Además, como mecanismo de recaudación y re integro, existe una compensación automática en formulario de IVA. Esto aplica para la declaración del IVA de exportadores habituales. Ellos al tener derecho a la devolución del impuesto percibido, tienen la posibilidad de compensarlo al momento de declarar.

En contraste, la compensación de créditos de tributos recaudados por personas naturales o jurídicas, no se admitirá cuando estas actúen como agentes de retención o de percepción. De igual manera, no serán admitidas las compensaciones de obligaciones adeudadas al Gobierno Nacional y demás entidades estatales, con títulos de la deuda pública externa.

Lo antes mencionado da la opción de acceder a un reembolso del IVA pagado. Esta opción es importante pues significaría un reingreso de rubros por pagos de este impuesto. Dependiendo del caso, aplicaría en determinados porcentajes de acuerdo al cumplimiento de los diferentes requisitos y parámetros que son solicitados por el ente de control para acceder a esta ventaja tributaria. Lo anterior permite la ley dentro de un ámbito de beneficios a los contribuyentes, incluyendo empresas del sector público, que es el sector de enfoque para este caso de estudio.

Respecto de la aplicación y manejo del IVA es necesario identificar el inicio de las obligaciones fiscales como ciudadano o persona jurídica en el país. Esto se lo ejecuta mediante un registro en el ente de control el Sistema de Renta Internas, SRI y a través del Registro Único del Contribuyente RUC.

En el documento que acredita el registro del RUC están las obligaciones tributarias que cada contribuyente debe cumplir y de acuerdo al giro del negocio estará obligado a presentar declaraciones de IVA mensual o semestral, lo primero cuando las compras y ventas sean con tarifa $12 \%$ y lo segundo cuando las solo ciertas compras graven tarifa $12 \%$ y las ventas sean siempre tarifa $0 \%$. 
La Normativa tributaria vigente, en cuanto a la declaración y pago del IVA señala los lineamientos para la declaración del impuesto, liquidación del impuesto y pago del impuesto respectivamente (Asamblea Constituyente Nacional, 2015).

En los casos que se asuma que un crédito tributario no podrá ser compensado durante los seis meses subsiguientes, el interesado podrá solicitar a la máxima autoridad del Servicio de Rentas Internas la compensación del crédito tributario por retenciones que hayan sido ejecutadas. Al no constituir pagos indebidos, las devoluciones 0 compensaciones de los saldos del IVA no causarán intereses.

En relación a las obligaciones de los contribuyentes, existen fechas máximas para la presentación y pago de sus declaraciones. Esto se realiza a través de un sistema que se instala a partir de los drivers entregados por el mismo SRI. Esto sirve de medio para el registro de los datos que entrega un archivo en formato $\mathrm{XML}$ que permite su carga a la plataforma del ente de control y este a su vez registra el cumplimiento de la obligación y emite el respectivo comprobante de pago de ser el caso y con esto finalizaría la obligación mensual y a posterior solo en casos especiales. Esto se detalla en los artículos 102 y 279 de la LORTI (2015).

Además de las declaraciones mensuales, se debe entregar el Informe de Cumplimiento Tributario (ICT). Este informe es un instrumento que enseña la opinión del auditor respecto al cumplimiento de las obligaciones fiscales del contribuyente sobre un período fiscal determinado. Para el efecto, se debe elaborar un conjunto de anexos establecidos por la administración tributaria en el cual se hace una conciliación tributaria entre lo declarado y los hallazgos del auditor. ( Rivera, Narváez, Giler y Erazo, 2019)

En cuanto a la gestión contable tributario, Vargas (2017), señala que deberá existir una cuenta contable en el pasivo que registre el IVA cobrado. De esto se entiende a posteriori deberá ser la cuanta que se debite cuando se haga el pago del impuesto. Por otra parte, el autor sostiene que debe existir una cuenta en el activo que permita registrar los pagos del IVA en las adquisiciones de bienes o servicios. Estas cuentas interactuarán haciendo su contabilización de forma contraria para la liquidación del impuesto para su posterior declaración y pago. 


\section{La carga tributaria y la liquidez}

Tal como se mencionó anteriormente, el IVA y otros impuestos tienen una afectación económica directa en la población. Por tanto, su grado de afectación ha sido explorado tanto en la carga tributaria como en la liquidez de las empresas.

Por un lado, la carga tributaria es el resultante de los valores pagados por concepto de impuestos, además de rubros que contablemente se registran como gastos por su naturaleza y movimiento (Alonso, Cantera, y Orozco, 2006). Por otro lado, la liquidez se ha definido como la capacidad que se tiene para hacer frente a las obligaciones, característica que tiene el dinero para ser aceptado como medio de pago (Villacis, 2008). En este sentido, cabe señalar que la carga tributaria, específicamente proveniente del pago del IVA, afecta a la liquidez de las empresas en distintos niveles (Nava y Venegas 2018). Además, al ser los impuestos ingresos permanentes para el Estado, estos son de carácter obligatorio y su pago es irremediable. Por lo tanto, estos pagos significan egresos de flujo de efectivo que afectan a la liquidez de los contribuyentes (Zapata, 2014). A lo anterior se suma que, a nivel empresarial, estos egresos no siempre son proyectados o planificados. La carencia de una planificación o guía tributaria representa un riesgo para una correcta gestión tributaria y el manejo financiero. Sin embargo, se sugiere que en muchos de los casos las empresas no contemplan o consideran necesario la planificación del pago de impuestos.

De aquí la necesidad de tener un lineamiento o guía que indique el manejo de los tributos a nivel empresarial. Se sugiere que esto contribuiría a evitar pagos innecesarios de multas y sanciones por incumplimiento de estas obligaciones.

Lo anterior fue ilustrado por Benítez (2016), el autor, a partir de revisiones de documentación de declaraciones y pagos de impuestos, pudo evidenciar que los desembolsos económicos realizados por pagos de tributos al ente del control de los impuestos eran elevados. Según Benítez esto genera atrasos en pagos de estas obligaciones y sanciones tributarias por diversos conceptos.

Además, los principales problemas que pueden presentarse por una falta de planificación y lineamientos tributarios son: carga tributaria alta, pagos excesivos de impuestos, 
endeudamientos por pagos de impuesto e incluso la evasión de impuestos por no tener flujo de efectivo.

\section{Impacto de la liquidez por pago de impuestos}

Tal como se ha mencionado anteriormente, el pago de impuestos es inevitable y el egreso de dinero es constante por este rubro. De acuerdo al IEGE (Instituto Europeo de Gestión Empresarial, 2007), este desembolso de dinero afectaría a los flujos de efectivo de una empresa, pues si además de no disponerlos tampoco se los ha planeado, el efecto será una disminución de la liquidez.

En este sentido, la necesidad de conocer cómo manejar la liquidez, cómo medirla y conocer su nivel óptimo es indispensable para evitar que ésta se vea afectada por desembolsos de obligaciones fiscales. Es decir, tomar en cuenta este particular a la hora de programar o planificar los flujos de efectivo.

En algunas circunstancias las personas jurídicas o personas naturales llegan a solicitar créditos bancarios para poder hacer la cancelación de sus obligaciones tributarias creando un efecto de endeudamiento en el contribuyente. Esto se debe principalmente a la falta de planificación, así como una guía tributaria.

\section{Liquidez}

Liquidez, según Torres y colegas (2018) se define como la posesión de efectivo o dinero líquido preciso en el momento adecuado, que nos permita hacer el pago de los compromisos contraídos. Los autores señalan que, mientas más fácil y rápido se puedan convertir los recursos del activo en dinero, dispondrá de mayor capacidad de pago para hacer frente a sus deudas y obligaciones.

De manera similar, Aguirre (2015) explica que la liquidez es la capacidad de tener efectivo en todo momento, el necesario para operar y hacer frente a todas las obligaciones adquiridas y tener la facilidad para efectuar su pago en el tiempo pactado o establecido. Sin embargo, es importante considerar también que la liquidez depende de otros factores como el tiempo requerido para convertir activos en dinero, la incertidumbre en el tiempo 
y del valor de la realización de los activos en dinero (Baena, 2008). El tomar en cuenta los factores antes mencionados incluye la planificación y programación de flujos de efectivo, haciendo que estos sean más ciertos y seguros.

Por lo antes expuesto, varias empresas se ven afectadas financiera $y$ administrativamente, especialmente en la liquidez (Kluwer, 2019). Es así que estas organizaciones solicitan créditos para poder cumplir con estas obligaciones, situación que podría presentarse tanto en la empresa pública como en la privada.

\section{Procedimientos tributarios}

La problemática expuesta anteriormente, sugiere la necesidad de establecer procedimientos que contribuyan al control y manejo de los impuestos.

Procedimiento, de acuerdo a la ISO 9000 (2005), es una forma especificada para llevar a cabo una actividad o un proceso. Kluwer (2019) define a los procedimientos tributarios como el conjunto de actividades para la administración, aplicación, gestión, e inspección de los impuestos. El autor asegura que estos juegan un papel importante en el cumplimiento de obligaciones tributarias y el ejercicio de derechos de los contribuyentes. Por otro lado, ha sido argumentado que la planificación de gestión tributaria pretende organizar los negocios o asuntos tributarios conforme a la Ley, con el fin de obtener ahorros tributarios (Massone, 2008). Adicionalmente, Zapata (2014) señala que la planificación tributaria contribuye al análisis de los caminos legalmente viables para incrementar las utilidades y para proporcionar directrices para administrar el flujo de efectivo resultante del ahorro tributario (Zapata, 2014).

En base a las definiciones anteriores, se postula que los procedimientos tributarios dan lineamientos para la consecución y apoyo de una planificación tributaria óptima y para un adecuado manejo de la carga fiscal. Esto soportado en la legislación local sin dejar de lado la tendencia global y las diferentes actualizaciones dentro del ámbito tributario aplicable. 


\section{Planificación tributaria en el sector público}

En relación a la injerencia de la planificación tributaria en la gestión pública, ha sido postulado que el objeto del trabajo directivo en el sector público es el de crear valor público en busca de un beneficio para la ciudadanía (Moore, 1998). Esto contrasta con el objeto del sector privado, que busca un valor privado y con intención de lucro.

Según la Ley Orgánica de Empresas Públicas (2017), empresas públicas son las instituciones que pertenecen al Estado. Estas se rigen a la Constitución de la República y cumplen con los siguientes atributos: son personas jurídicas con derecho público, cuentan con su propio patrimonio, tienen autonomía financiera, económica, administrativa y de gestión.

Estas empresas, de acuerdo a la legislación nacional, están encaminadas a la gestión de sectores estratégicos del país, a prestar servicios públicos, manejando y aprovechando de manera sustentable de recursos naturales y bienes públicos $y$, al desarrollo de actividades económicas que corresponden al Estado.

Adicionalmente, al ser estas empresas públicas responsables de la gestión de sectores estratégicos y tener que responder ante el Estado y éste ante sus mandantes, la información revelada sobre el uso y manejo de los recursos públicos debe ser clara y confiable. La planeación tributaria, por tanto, puede ser considerada como un medio para el logro de los objetivos institucionales públicos, que coadyuvan a conocer la realidad fiscal y los compromisos que de ella derivan (Bedoya, 2011).

\section{METODOLOGÍA}

El tipo de investigación fue descriptiva dado que se expusieron características propias de los procedimientos tributarios para el tratamiento del IVA en una empresa pública. Como unidad de analisis se consideró a la Empresa FARMASOL EP., y como universo de estudio a cuatro actores clave de la empresa: Gerente, Subgerente Administrativo Financiero, Contador y Tesorero (Hernández, Fernández y Baptista, 2014). 


\section{RESULTADOS}

A continuación se presentan los principales resultados identificados en la fase de diagnostico:

a) Actualmente no realizan mediciones de liquidez ni se calcula la liquidez circulante en FARMASOL EP.

b) Se puede evidenciar que la empresa no cuenta con un departamento de recuperación de cartera.

c) La empresa cuenta con políticas para pagos a proveedores los cuales son depositados oportunamente.

d) Se ha podido constatar que no se realizan estudios o análisis de flujo de efectivo Farmasol EP.

e) Se evidencia que la empresa cuenta con fondos necesarios para cumplir con sus obligaciones tributarias.

A partir de los hallazgos mencionados anteriormente, se pudo evidenciar que la definición de procedimientos tributarios para manejo del IVA mejoraría la liquidez de la empresa, así como también la gestión tributaria interna.

Luego de lo presentado anteriormente, se procedió con la revision de documentos para validar la informacion recolectada.

En esta actividad, se pudo evidenciar que FARMASOL EP no cuenta con procedimientos tributarios para la administración del IVA. Por tanto, dicha gestion se ha realizado de manera informal y heterogenea. Por ejemplo, la empresa compra y vende gravando este impuesto y los desembolsos para cumplir con la obligacion del pago por el impuesto en mención.

Se pudo evidenciar también, que al no contar con procedmientos para la gestion del IVA, los pagos por este concepto estan afectando a la liquidez de la empresa.

Finalmente, se pudo constatar que en en la empresa el IVA no compensado, producto del factor de proporcionalidad, se registra en una cuenta de gasto, lo que está afectando a las utilidades. 


\section{Procedimientos Tributarios}

En base a la información recolectada y a los resultados expuestos anteriormente, se han planteado procedimientos tributarios que se espera cubran las principales aristas de la gestión tributaria:

1. Presupuesto de inventarios y compras Bienes y Servicios con tarifa $12 \%$.

2. Presupuesto de ventas de Bienes y Servicios con tarifa $12 \%$.

3. Presupuesto de pago de impuestos.

4. Flujos de caja proyectados.

5. Declaración del IVA mensual.

6. Elaboración de anexo transaccional.

Procedimiento 1.

\begin{tabular}{|l|l|l|l|}
\hline & \multicolumn{2}{|c|}{$\begin{array}{c}\text { Procedimiento: Presupuesto de } \\
\text { Inventarios y Compras de Bienes y y } \\
\text { Servicios con tarifa 12\% }\end{array}$} & $\begin{array}{l}\text { Versión: 1 } \\
\text { Fecha: 21/08/2019 } \\
\text { Código: }\end{array}$ \\
\hline $\begin{array}{l}\text { Objetivo: Elaborar un presupuesto de de } \\
\text { compras de inventario y de compras de otros } \\
\text { bienes y servicios (gasto corriente) gravados } \\
\text { con tarifa 12\% para establecer con antelación } \\
\text { el IVA a pagar en compras y cuanto de este } \\
\text { podrá ser usado como crédito tributario. }\end{array}$ & $\begin{array}{l}\text { Alcancen las actividades y } \\
\text { responsabilidades para el Presupuesto de } \\
\text { Ários y Compras B\&S con tarifa 12\% }\end{array}$ \\
\hline Proceso: Gestión Financiera & Subproceso: Gestión Presupuestaria \\
\hline
\end{tabular}




\section{Responsables: Analista de}

Abastecimiento y Analista de

Adquisiciones:

1. Levanta mensualmente las necesidades de compra de acuerdo al buffer. ${ }^{1}$

2. Realiza un cuadro comparativo de los todos los ítems a comprar.

3. Establece un presupuesto de compras.

4. Clasifica los ítems gravados con tarifa $12 \%$ y tarifa $0 \%$.

Subgerente Logístico:

5. Convoca a una reunión al inicio de cada mes para establecer las compras del periodo en mención a todo el departamento de abastecimiento.

6. Recopila toda la información sobre los presupuestos de compras de todos los analistas y la envía al Jefe Financiero.

\section{Jefe Financiero:}

7. Procesa la información del presupuesto de Compras y el presupuesto de Ventas para establecer el rubro que se tendrá por IVA pagado.

8. Revisa y asegura la disponibilidad de flujo en base a las necesidades presupuestadas.

9. Establece estrategias para el período si es que lo requiere y comunica al Subgerente Administrativo Financiero.
Diagrama:

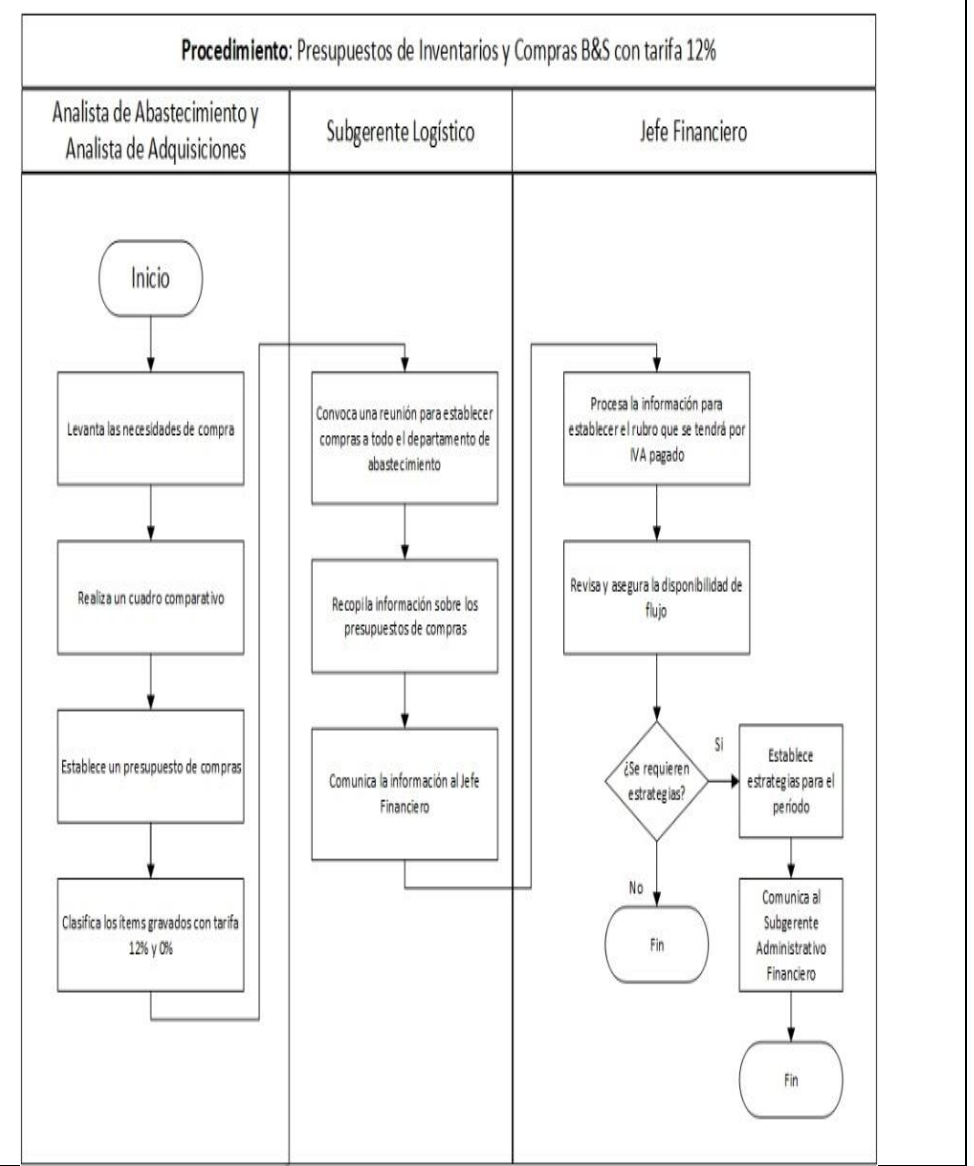

\section{Procedimiento 2.}

\section{formesel}

\section{Procedimiento: Presupuesto de Ventas de Bienes y Servicios con tarifa $12 \%$}

Versión: 1

Fecha: $21 / 8 / 2019$

Código:
Objetivo: Elaborar un presupuesto de ventas de bienes y servicios gravados con tarifa $12 \%$ para establecer con antelación que valor se va a recaudar como IVA cobrado y cuanto de este se compensará como el crédito tributario en compras para poder establecer el IVA a pagar y cuando se convertirá en IVA no compensado.
Alcance: Definir las actividades y responsabilidades para el Presupuesto de Ventas de B\&S con tarifa $12 \%$

Área: Comercial

\footnotetext{
${ }^{1}$ Algoritmo recursivo de la gestión de inventarios que sirve para determinar el nivel óptimo o de seguridad en la cantidad de los ítems o productos en bodegas, tiendas o puntos de venta, (Heredia , 2013).
} 
Proceso: Gestión Financiera

\section{Responsables:}

\section{Supervisor de ventas:}

1. Elabora los prepuestos de ventas en base de históricos por cada punto de venta.

2. Establece la proporción que corresponde a ventas gravadas con tarifa $12 \%$ y tarifa $0 \%$.

3. Estructura el presupuesto de ventas clasificado por este criterio.

\section{Subgerencia Comercial:}

4. Convoca a una reunión al inicio de cada mes para establecer el presupuesto de ventas.

5. Recopila información sobre los presupuestos de ventas y la envía al Jefe Financiero.

\section{Jefe Financiero:}

6. Procesa la información del presupuesto de Compras y el presupuesto de Ventas para establecer el rubro que se tendrá por IVA cobrado.

7. Revisa y asegura la disponibilidad de flujo en base a las necesidades presupuestadas.

8. Establece estrategias para el período si es que lo requiere y comunica al Subgerente Administrativo Financiero.

\section{Diagrama:}

\section{Subproceso: Gestión Presupuestaria}

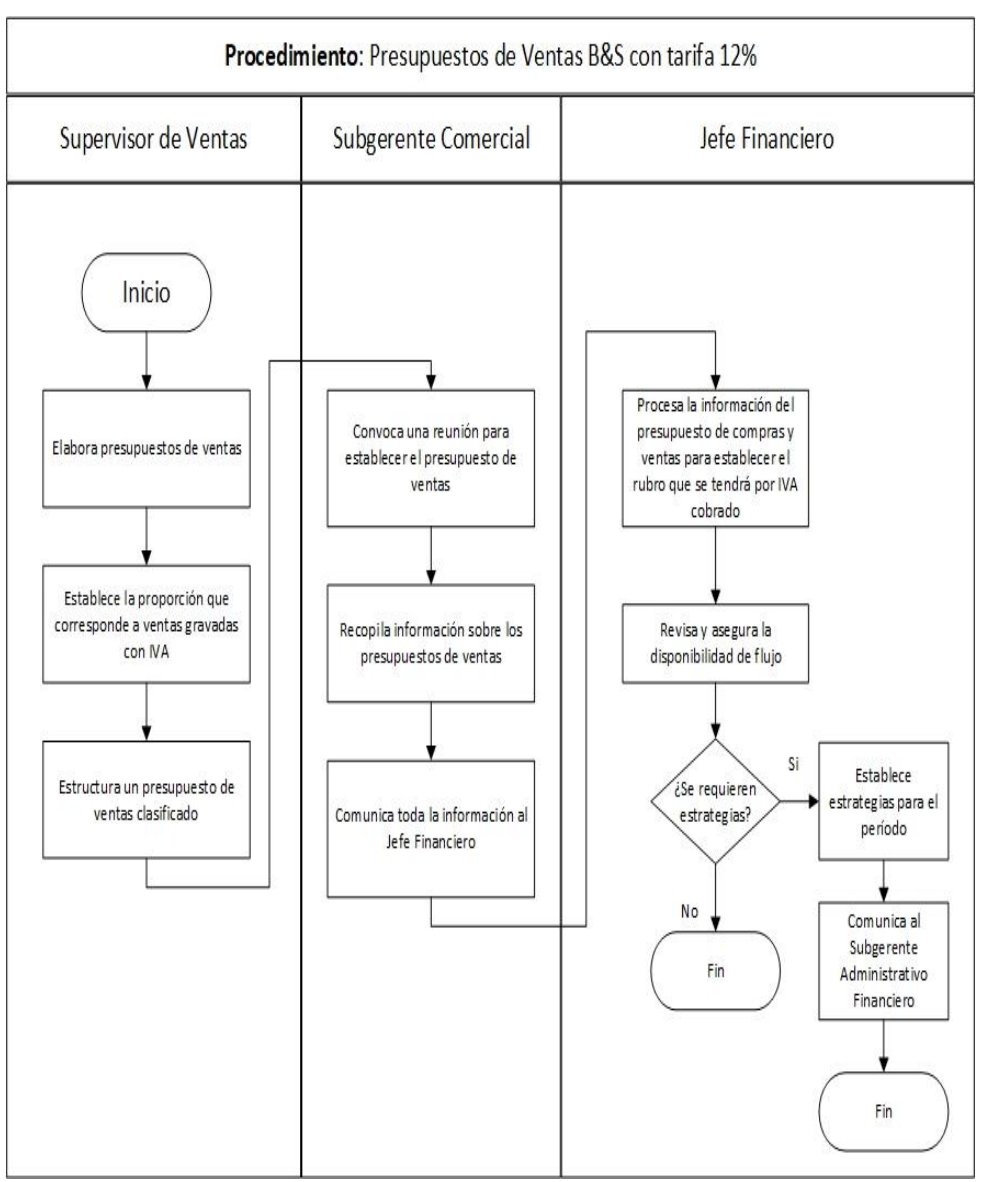

\section{Procedimiento 3.}

\begin{tabular}{|c|c|l|}
\hline formose & $\begin{array}{c}\text { Procedimiento: Presupuesto de Pago } \\
\text { de Impuestos }\end{array}$ & $\begin{array}{l}\text { Versión: } 1 \\
\text { Fecha: 21/8/2019 } \\
\text { Código: }\end{array}$ \\
\hline
\end{tabular}

Procedimiento 4. 


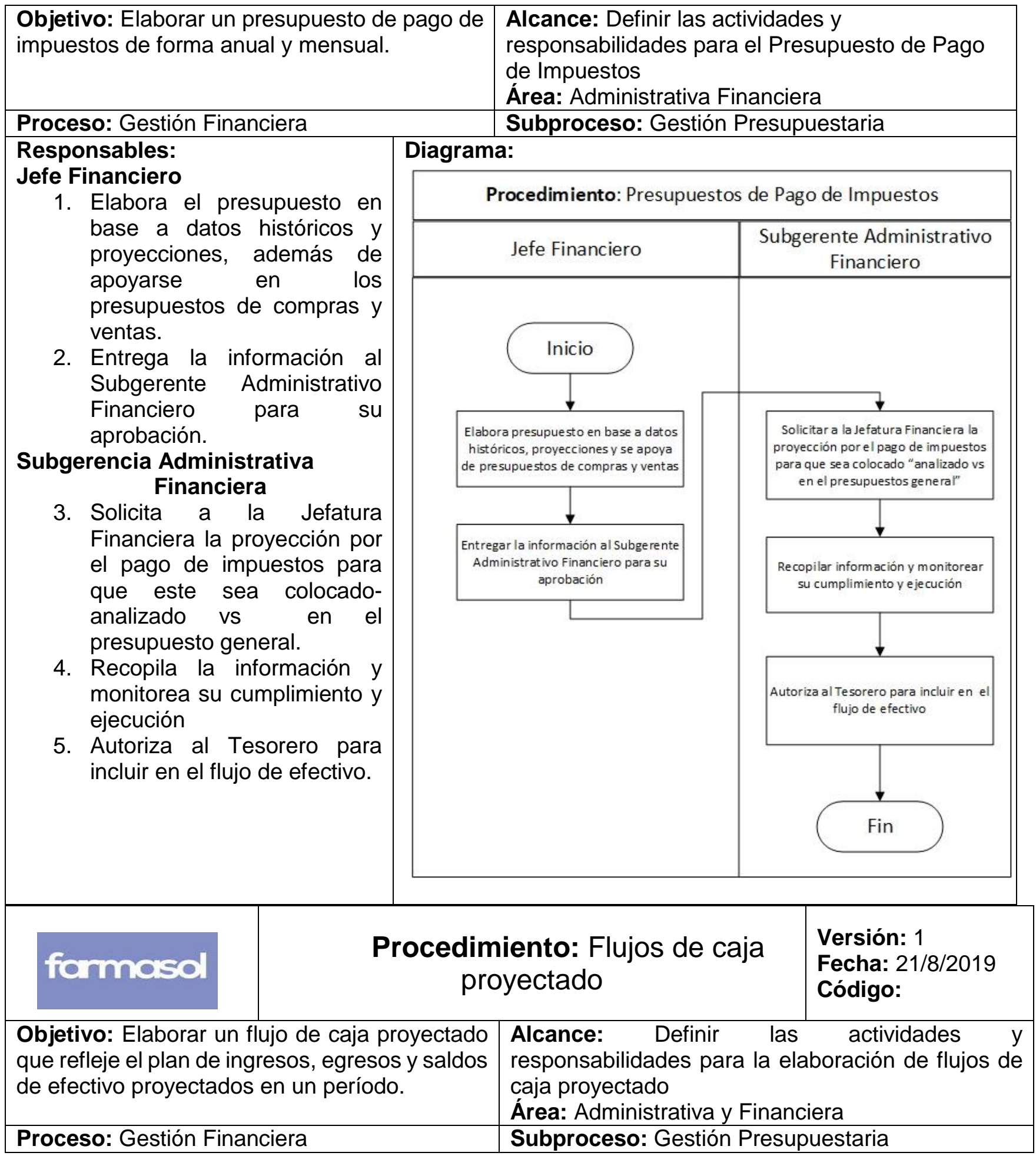




\section{Responsables:}

\section{Tesorero}

1. Revisa el estado de resultados del mes anterior para observar las cuentas o rubros que generan ingresos y egresos.

2. Revisa el estado de situación financiera del mes anterior para observar las cuentas o rubros que evidencian incrementos decrementos de un periodo a otro.

3. Revisa los presupuestos de compras y ventas para el periodo en cuestión.

4. Recopila la información y se la plasma en un formato clasificando ingresos y gastos.

5. Registra todos los rubros y cuentas que se consideran entradas de dinero en una columna de ingresos.

6. Registra todos los rubros y cuentas que se consideran salidas de dinero en una columna de egresos.

7. Totaliza los ingresos y egresos y se determina superávit o déficit.

8. Envía al Jefe Financiero Jefe Financiero

9. $\mathrm{Si}$ es superávit analiza la pertenencia de obtenerlo, generar inversiones o cubrir obligaciones (estrategias financieras)

10. Si es déficit analiza opciones de ingresos 0 se considere la posibilidad de postergar pagos (estrategias financieras)

11. Se autoriza y aplica

\section{Diagrama:}

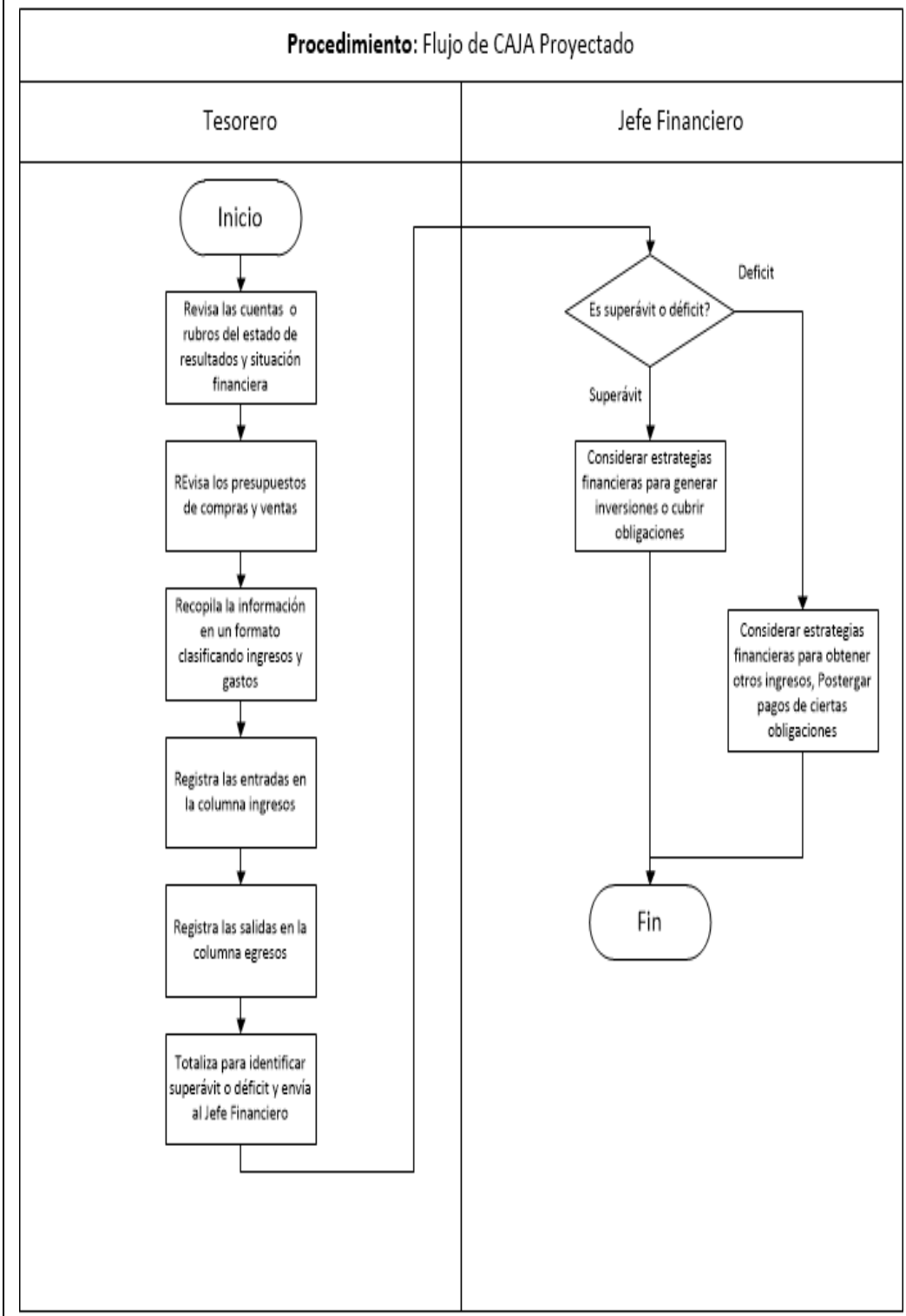

Procedimiento 5. 


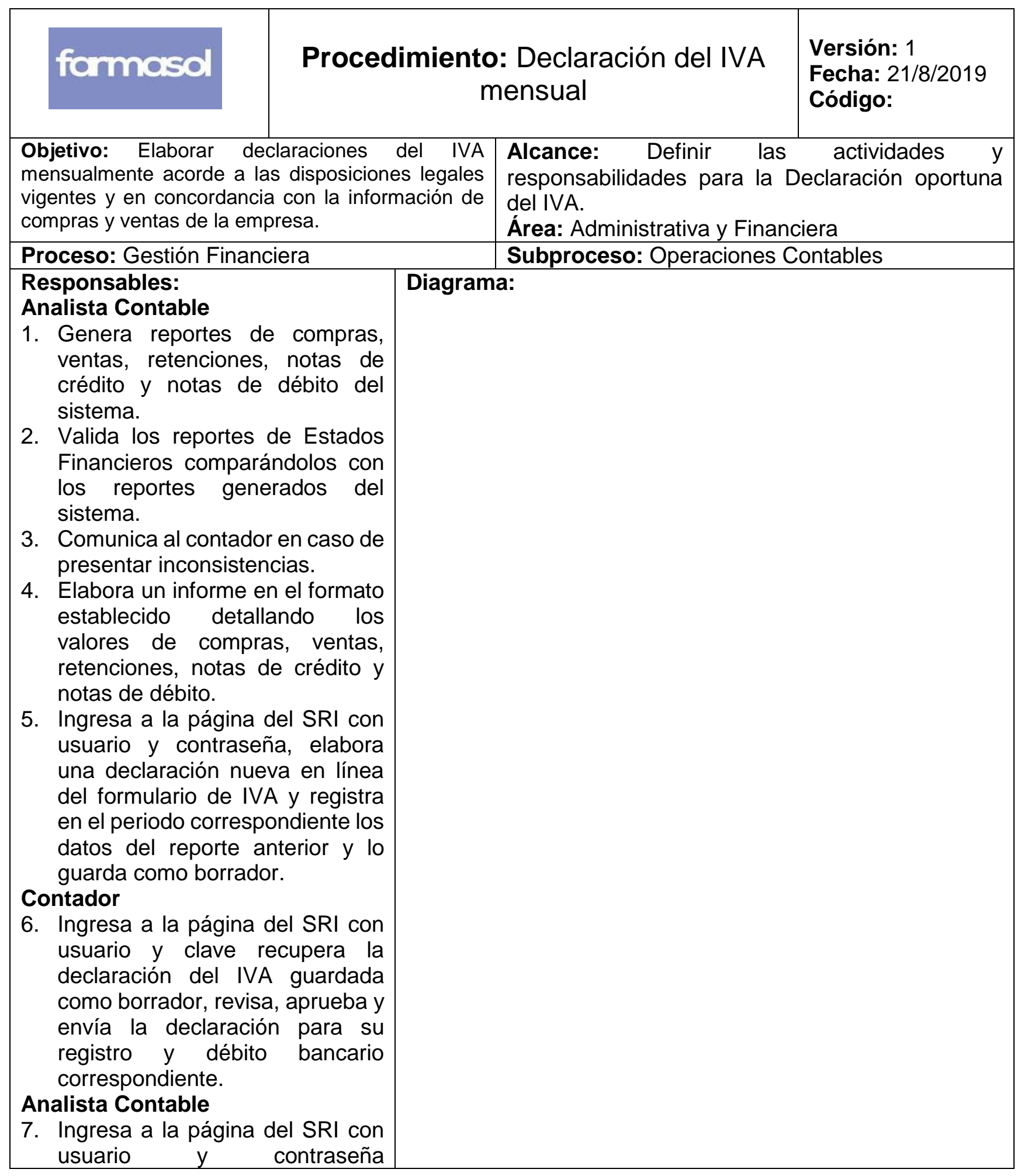




\section{proporcionados y cargar los} archivos generados.

8. Imprime los comprobantes de declaraciones de formularios $y$ archivar.

\section{Procedimiento 6.}

\section{Procedimiento: Elaboración de anexo transaccional}




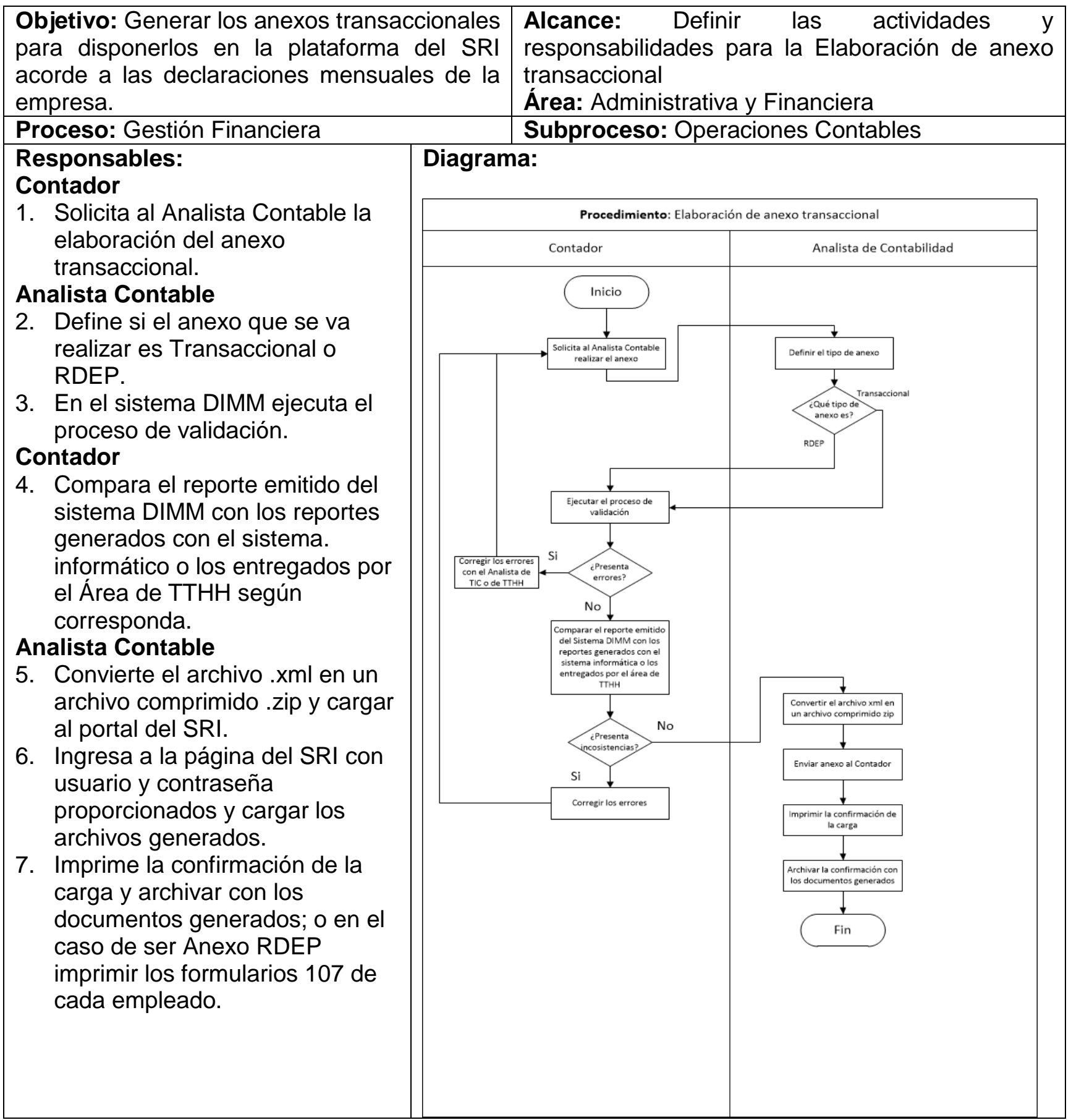

\section{DISCUSIÓN}


De la revisión literaria se concluye que los tributos juegan un papel vital para las economías de las personas, las organizaciones y los países. De estos, el pago y administración tributaria del IVA puede impactar positiva o negativamente a la liquidez y utilidades de las empresas. Esto dependiendo de si la gestión tributaria se realiza mediante lineamientos o procedimientos tributarios acordes a la ley. Por tal razón, la definición de dichos procedimientos para el pago del IVA se considera necesario.

En cuanto al caso de estudio, los resultados indican que Farmasol EP actualmente no sigue lineamientos formales en relacion a la planificacion y gestion tributaria del IVA. De esto, se pudo inferir que procedimientos tributarios encaminados a esta debilidad pueden aportar a una mejora en los flujos de efectivo, y eventualmente en la liquidez.

A partir de lo anterior, se definieron y expusieron procedimientos tributarios para el manejo del IVA en Farmasol EP. De los procedimientos propuestos se concluye que estos cubren los principales aspectos para la gestion del IVA en la empresa. Siguiendo el concepto de procedimiento documentado, con responsabilidades especificas y actividades por cargo.

De lo anterior, se espera una mejora en la gestion tributaria del IVA en Farmasol EP. Consecuentemente, se espera también un impacto positivo en la liquidez como en las utilidades de Farmasol.

\section{REFERENCIAS CONSULTADAS}

1. Alonso, J., Cantera, M., \& Orozco, B. (2006). SECTOR PÚBLICO Y DÉFICIT FISCAL. Valle del Cauca: Departamento de Economía - Universidad ICESI.

2. Bedoya, O. (2011). La incidencia de la planeación tributaria en el crecimiento y logro de los objetivos del ente economico. VISIÓN CONTABLE, 136.

3. Benitez, J. (2016). Incidencia de las obligaciones tributarias en las utilidades de MIPYMES en Ecuador. Revista Caribeña de Ciencias Sociales, 2.

4. Buena, N. (Septiembre de 2008). Comision Nacional del Mercado de Valores. Obtenido 
5. ClústerFarma. (02 de 06 de 2017). Obtenido de ClústerFarma: http://farma.ebizor.com/ecuador-como-productor-farmaceutico/

6. ClústerFarma. (02 de 06 de 2017). Obtenido de ClústerFarma: http://farma.ebizor.com/ecuador-como-productor-farmaceutico/

7. Heredia. N. L. (2013). Gerencia de compras: La nueva estrategia competitiva. Bogotá: ECOE EDICIONES.

8. Hernández, Fernández y Baptista (2014). Metodología de la investigación. México, Mc Graw Hill Hispanoamericana. Hill Internacional

9. Hidalgo Vanegas, J. C., Narváez Zurita, C. I., \& Erazo Álvarez, J. C. (2019). Devolución del crédito fiscal del impuesto al valor agregado (IVA) al sector exportador bananero en el Ecuador. 593 Digital Publisher CEIT, 10. Instituto Europeo de Gestión Empresarial. (2007).

10. Larraín, F., \& Sachs, J. (2002). Macroeconomía en la economía global. Buenos Aires: Pearson Education S.A.

11.Lìderes. (16 de 03 de 2019). Obtenido de Lìderes: https://www.revistalideres.ec/lideres/ordenan-empresas-publicas.html

12. Massone, M. (2008). Elusión y Planificación Tributaria. Santiago de Chile: Alessandri \& Compañía.

13. Martínez Prats, G. (2019). Análisis del Sistema de Estadística de la Balanza Comercial de México. Revista Arbitrada Interdisciplinaria Koinonía, 4(7), 13-32. doi:http://dx.doi.org/10.35381/r.k.v4i7.192

14. Moore, M. (1998). Gestión estratégica y Creación de Valor en el Sector Público. Barcelona: Paidós Ibérica S.A.

15. Paz, J. J., \& Cepeda, M. (2015). Historia de los impuestos en Ecuador. Quito: SRITHE PUCE.

16. Rivera Ríos, K. M., Narváez Zurita, C. I., Giler Escandón, L. V., \& Erazo Álvarez, J. C. (2019). Evaluación tributaria de los gastos imputables para la declaración del Impuesto al Valor Agregado IVA en la Asociación de servicios de alimentación, limpieza y mensajería La unión hace la fuerza "ASOUNF". 593 Digital Publisher CEIT, 68. 
17. Say, J. (1983). Tratado de Economia Politica. Sao Paulo: Victor Civita.

18. Seitz, M. (27 de Julio de 2015). http://www.bbc.com. Obtenido de http://www.bbc.com/mundo/video fotos/2015/07/150722 economia america lati na iva consumidores lista ms

19. Sistema de Rentas Internas. (17 de Junio de 2018). SRI. Obtenido de http://www.sri.gob.ec/web/guest/que-es-el-sri

20.Torres, T., Riv, R., \& Ortiz, A. (23 de Junio de 2018). Barcelona School of Management.

Obtenido

de https://www.barcelonaschoolofmanagement.upf.edu/documents/Tesina-Lagestion-de-la-liquidez.pdf

21. Vargas, C. (2017). Contabilidad Tributaria. Bogotá: ECOE Ediciones.

22. Vatlive. (17 de Junio de 2018). VATlive. Obtenido de https://www.vatlive.com/vatrates/?sessionld=1529247143908\&referrer=\&lastReferrer=www.vatlive.com

23. Wolters Kluwer España, S.A. (2019). Practica fiscal para abogados. Madrid: Wolters Kluwer España, S.A.

24.Zapata, J. (2014). La planificación tributaria como mecanismo para lograr la eficiencia económica en el tratamiento del impuesto a la renta de las sociedades dedicadas al turismo receptivo en el Ecuador. Quito, Pichincha.

\section{REFERNCES CONSULTED}

1. Alonso, J., Cantera, M., \& Orozco, B. (2006). PUBLIC SECTOR AND TAX DEFICIT. Valle del Cauca: Department of Economics - ICESI University.

2. Bedoya, O. (2011). The incidence of tax planning on the growth and achievement of the objectives of the economic entity. ACCOUNTING VISION, 136.

3. Benitez, J. (2016). Incidence of tax obligations in the profits of MSMEs in Ecuador. Caribbean Journal of Social Sciences, 2.

4. Buena, N. (September 2008). National Stock Market Commission. Retrieved from https://www.cnmv.es/docportal/publicaciones/monografias/mon2008 32.pdf 
5. Cluster Signature. (02 of 06 of 2017). Obtained from ClústerFarma: http://farma.ebizor.com/ecuador-como-productor-farmaceutico/

6. Cluster Signature. (02 of 06 of 2017). Obtained from ClústerFarma: http://farma.ebizor.com/ecuador-como-productor-farmaceutico/

7. Heredia. N. L. (2013). Purchasing Management: The new competitive strategy. Bogotá: ECOE EDITIONS.

8. Hernández, Fernández and Baptista (2014). Investigation methodology. Mexico, Mc Graw Hill Hispanic American. Hill International

9. Hidalgo Vanegas, J. C., Narváez Zurita, C. I., \& Erazo Álvarez, J. C. (2019). Return of the tax credit of the value added tax (VAT) to the banana export sector in Ecuador. 593 Digital Publisher CEIT, 10.

10. European Institute of Business Management. (2007).

11. Larraín, F., \& Sachs, J. (2002). Macroeconomy in the global economy. Buenos Aires: Pearson Education S.A.

12. Leaders. (16 of 03 of 2019). Obtained from Leaders: https://www.revistalideres.ec/lideres/ordenan-empresas-publicas.html

13. Massone, M. (2008). Avoidance and Tax Planning. Santiago, Chile: Alessandri \& Company.

14. Martínez Prats, G. (2019). Analysis of the Statistical System of the Trade Balance of Mexico. Interdisciplinary Arbitrated Review Koinonía, 4 (7), 13-32. doi: http: //dx.doi.org/10.35381/r.k.v4i7.192

15. Moore, M. (1998). Strategic Management and Value Creation in the Public Sector. Barcelona: Paidós Ibérica S.A.

16.Paz, J. J., \& Cepeda, M. (2015). History of taxes in Ecuador. Quito: SRI-THE PUCE.

17. Rivera Ríos, K. M., Narváez Zurita, C. I., Giler Escandón, L. V., \& Erazo Álvarez, J. C. (2019). Tax assessment of the expenses attributable to the declaration of VAT Value Added Tax in the Association of food, cleaning and courier services The union makes the force "ASOUNF". 593 Digital Publisher CEIT, 68.

18. Say, J. (1983). Treaty of Political Economy. Sao Paulo: Victor Civita. 
19. Seitz, M. (July 27, 2015). http://www.bbc.com Retrieved from http://www.bbc.com/mundo/video fotos/2015/07/150722 economia america lati na iva consumidores lista $\mathrm{ms}$

20. Internal Revenue System. (June 17, 2018). SRI Retrieved from http://www.sri.gob.ec/web/guest/que-es-el-sri

21. Torres, T., Riv, R., \& Ortiz, A. (June 23, 2018). Barcelona School of Management. Obtained from https://www.barcelonaschoolofmanagement.upf.edu/documents/Tesina-Lagestion-de-la-liquidez.pdf

22. Vargas, C. (2017). Tax Accounting Bogotá: ECOE Editions.

23. Vatlive. (June 17, 2018). VATlive. Retrieved from https://www.vatlive.com/vatrates/?sessionld=1529247143908\&referrer=\&lastReferrer=www.vatlive.com

24. Wolters Kluwer España, S.A. (2019). Tax practice for lawyers. Madrid: Wolters Kluwer España, S.A.

25.Zapata, J. (2014). Tax planning as a mechanism to achieve economic efficiency in the treatment of income tax of societies dedicated to inbound tourism in Ecuador. Quito, Pichincha. 